\title{
Clinical characteristics and long-term outcomes in patients with ruptured posterior inferior cerebellar artery aneurysms: a comparative analysis
}

\author{
Richard W. Williamson, MD, David A. Wilson, MD, Adib A. Abla, MD, Cameron G. McDougall, MD, \\ Peter Nakaji, MD, Felipe C. Albuquerque, MD, and Robert F. Spetzler, MD
}

Division of Neurological Surgery, Barrow Neurological Institute, St. Joseph's Hospital and Medical Center, Phoenix, Arizona

OBJECT Subarachnoid hemorrhage (SAH) from ruptured posterior inferior cerebellar artery (PICA) aneurysms is uncommon, and long-term outcome data for patients who have suffered such hemorrhages is lacking. This study investigated in-hospital and long-term clinical data from a prospective cohort of patients with SAH from ruptured PICA aneurysms enrolled in a randomized trial; their outcomes were compared with those of SAH patients who were treated for other types of ruptured intracranial aneurysms. The authors hypothesize that PICA patients fare worse than those with aneurysms in other locations and this difference is related to the high rate of lower cranial nerve dysfunction in PICA patients.

METHODS The authors analyzed data for 472 patients enrolled in the Barrow Ruptured Aneurysm Trial (BRAT) and retrospectively reviewed vasospasm data not collected prospectively. In the initial cohort, 57 patients were considered angiographically negative for aneurysmal SAH source and did not receive treatment for aneurysms, leaving 415 patients with aneurysmal SAH.

RESULTS Of 415 patients with aneurysmal SAH, 22 (5.3\%) harbored a ruptured PICA aneurysm. Eight of them had dissecting/fusiform-type aneurysms while 14 had saccular-type aneurysms. Nineteen PICA patients were treated with clipping (1 crossover from coiling), 2 were treated with coiling, and 1 died before treatment. When comparing PICA patients to all other aneurysm patients in the study cohort, there were no statistically significant differences in age (mean $57.6 \pm$ 11.8 vs $53.9 \pm 11.8$ years, $p=0.17$ ), Hunt and Hess grade median III [IQR II-IV] vs III [IQR II-III], $p=0.15)$, Fisher grade median 3 [IQR 3-3] vs 3 [IQR 3-3], $p=0.53$ ), aneurysm size (mean $6.2 \pm 3.0$ vs $6.7 \pm 4.0 \mathrm{~mm}, p=0.55$ ), radiographic vasospasm ( $53 \%$ vs $50 \%, p=0.88)$, or clinical vasospasm ( $12 \%$ vs $23 \%, p=0.38)$. PICA patients were more likely to have a fusiform aneurysm (36\% vs $12 \%, p=0.004)$ and had a higher incidence of lower cranial nerve dysfunction and higher rate of tracheostomy/percutaneous endoscopic gastrostomy placement compared with non-PICA patients $(50 \%$ vs $16 \%, p<0.001)$. PICA patients had a significantly higher incidence of poor outcome at discharge $(91 \%$ vs $67 \%, p=$ 0.017 ), 1 -year follow-up (63\% vs $29 \%, p=0.002$ ), and 3 -year follow-up ( $63 \%$ vs $32 \%, p=0.006$ ).

CONCLUSIONS Patients with ruptured PICA aneurysms had a similar rate of radiographic vasospasm, equivalent admission Fisher grade and Hunt and Hess scores, but poorer clinical outcomes at discharge and at 1- and 3-year followup when compared with the rest of the BRAT SAH patients with ruptured aneurysms. The PICA's location at the medulla and the resultant high rate of lower cranial nerve dysfunction may play a role in the poor outcome for these patients. Furthermore, PICA aneurysms were more likely to be fusiform than saccular, compared with non-PICA aneurysms; the complex nature of these aneurysms may also contribute to their poorer outcome.

http://thejns.org/doi/abs/10.3171/2014.10.JNS141079

KEY WORDS PICA; aneurysm; subarachnoid hemorrhage; outcomes; vascular disorders

ABBREVIATIONS BRAT = Barrow Ruptured Aneurysm Trial; GOS = Glasgow Outcome Scale; ICU = intensive care unit; IQR = interquartile range; $\mathrm{mRS}=$ modified Rankin Scale; $P E G$ = percutaneous endoscopic gastrostomy; $\mathrm{PICA}=$ posterior inferior cerebellar artery; $\mathrm{SAH}$ = subarachnoid hemorrhage.

SUBMITTED May 14, 2014. ACCEPTED October 20, 2014.

INCLUDE WHEN CITING Published online April 17, 2015; DOI: 10.3171/2014.10.JNS141079.

DISCLOSURE The authors report no conflict of interest concerning the materials or methods used in this study or the findings specified in this paper. 
A NEURYSMS of the posterior inferior cerebellar artery (PICA) are relatively rare, comprising approximately $0.5 \%-3 \%$ of all intracranial aneurysms. ${ }^{10,11}$, 14,15 The majority of patients with these aneurysms present with a subarachnoid hemorrhage (SAH), although patients can occasionally present with symptoms secondary to mass effect on the brainstem or lower cranial nerves. ${ }^{15}$ The location of the PICAs next to the medulla and lower cranial nerves can make treatment challenging, especially in the face of SAH. ${ }^{7,16}$ Both microsurgical and endovascular treatment options exist, with acceptable rates of aneurysm occlusion; however, long-term outcome data are lacking for SAH patients with ruptured PICA aneurysms..$^{2-6,13,16}$ In this study, we aim to better define the clinical nuances, complications, and long-term outcomes of ruptured PICA aneurysms in patients who were treated as part of the Barrow Ruptured Aneurysm Trial (BRAT) and compare the clinical characteristics of those with ruptured PICA aneurysms versus those with all other types of ruptured intracranial aneurysms. ${ }^{12}$ We hypothesize that patients with ruptured PICA aneurysms fare worse than those with ruptured aneurysms in other locations and that the high rate of lower cranial nerve dysfunction may help to explain this worse outcome.

\section{Methods \\ Patient Population}

Between March 2003 and January 2007, 725 patients with SAH were screened for inclusion as part of the BRAT, and 472 patients were included in analyses as part of that study. Of the 472 patients enrolled, 57 were considered angiographically negative with no identifiable source for SAH and did not receive aneurysm treatment, leaving 415 patients with aneurysmal SAH. Prospectively collected data included age, sex, medical comorbidities, Hunt and Hess grade and Fisher grade at admission, treatment modality (clip ligation vs coil embolization), other surgical procedures, complications, and modified Rankin Scale (mRS) score at discharge and at the 1-year and 3-year follow-up evaluations. Angiographic and clinical vasospasm data, which were not collected during the study period, were collected retrospectively. All radiographic data were evaluated and interpreted by an independent neuroradiologist.

\section{Treatment}

All patients were admitted to the neurological intensive care unit (ICU) with external ventricular drainage as necessary. Patients were evaluated preoperatively with either conventional digital subtraction angiography or computed tomography angiography. Patients with a PICA aneurysm assigned to microsurgical clipping underwent a standard far-lateral craniotomy. Saccular aneurysms were treated with primary clip occlusion when possible. Dissecting and/or distal PICA aneurysms were treated with clip reconstruction, clip wrapping, or aneurysm trapping with bypass, as determined by the treating surgeon. Patients assigned to endovascular occlusion were treated with primary coil occlusion without stent placement.

All patients underwent standard SAH treatment in the
ICU, which included nimodipine and transcranial Doppler ultrasound screening. Triple-H therapy (hypertension, hypervolemia, and hemodilution) was used at the discretion of the attending surgeon to manage radiographic and/ or clinical vasospasm. In the majority of cases, cerebral digital subtraction angiography was performed 7 days after ictus to screen for vasospasm. The presence of radiographic vasospasm was determined by an independent neuroradiologist. Clinical vasospasm was defined either as patients having neurological examination findings concurrent with radiographic vasospasm in the affected vascular territory or as patients having radiographic vasospasm who responded to triple- $\mathrm{H}$ therapy with resolution of their symptoms. Once out of the vasospasm window, patients were weaned off their external ventricular drains when possible. Ventriculoperitoneal shunts were placed for patients with persistent communicating hydrocephalus. Patients with persistent lower cranial nerve dysfunction and swallowing difficulties were treated with percutaneous tracheostomy and/or percutaneous endoscopic gastrostomy (PEG) tube placement.

\section{Statistical Analysis}

Data from patients with PICA and non-PICA aneurysms were compared using independent t-tests for patient age and aneurysm size, chi-square test for sex, MannWhitney U-test for Hunt and Hess and Fisher grades, and Z-test of proportions for vasospasm. An mRS score greater than 2 was considered a poor outcome. A p value of less than 0.05 was considered significant.

\section{Results}

Of 415 patients with aneurysmal SAH, 22 (5.3\%) harbored PICA aneurysms. Eighteen patients were assigned to microsurgical clipping, 3 were assigned to endovascular coil occlusion (1 of whom crossed over to clipping), and 1 died before treatment assignment. Eleven patients were male and 11 were female. The 22 patients' mean age ( \pm SD) was $57.6 \pm 11.8$ years. The median admission Hunt and Hess grade for patients with PICA aneurysms was III (interquartile range [IQR] II-IV), and the median admission Fisher grade was 3 (IQR 3-3). The mean aneurysm size was $6.2 \pm 3.0 \mathrm{~mm}$. A higher percentage of patients with PICA aneurysms were male compared with patients with aneurysms in other locations. In addition, patients with PICA aneurysms were more likely to have a dissecting fusiform aneurysm compared with patients with nonPICA aneurysms (36\% vs $12 \%$, respectively, $\mathrm{p}=0.004)$ (Table 1).

Patients treated with microsurgical clipping underwent a standard far-lateral craniotomy. The 8 patients with dissecting fusiform-type aneurysms were treated surgically with a variety of techniques to secure the aneurysm: 5 were treated with aneurysm wrapping and clip reconstruction, 1 was treated with occipital artery-PICA bypass and aneurysm resection, and 1 was treated with aneurysm resection and PICA-PICA reimplantation. One patient had an SAH secondary to a dissecting PICA aneurysm that was proximal to a small cerebellar arteriovenous malformation. This aneurysm was treated with clip reconstruc- 
TABLE 1. Patient and aneurysm characteristics stratified by aneurysm location

\begin{tabular}{|c|c|c|c|}
\hline Characteristic & $\begin{array}{c}\text { PICA } \\
\text { Aneurysm }\end{array}$ & $\begin{array}{l}\text { Non-PICA } \\
\text { Aneurysm }\end{array}$ & $p$ Value \\
\hline No. of pts & 22 & 393 & \\
\hline Mean age in yrs $( \pm S D)$ & $57.6 \pm 11.8$ & $53.9 \pm 11.8$ & 0.17 \\
\hline No. of female pts & $11(50 \%)$ & $282(72 \%)$ & 0.03 \\
\hline Hunt \& Hess grade & & & 0.15 \\
\hline Median & III & III & \\
\hline Interquartile range & II-IV & $\|-\| \|$ & \\
\hline Fisher grade (range) & & & 0.53 \\
\hline Median & 3 & 3 & \\
\hline Interquartile range & $3-3$ & $3-3$ & \\
\hline $\begin{array}{l}\text { Mean aneurysm size in } \mathrm{mm} \\
( \pm \mathrm{SD})\end{array}$ & $6.2 \pm 3.0$ & $6.7 \pm 4.0$ & 0.55 \\
\hline $\begin{array}{l}\text { No. of dissecting fusiform } \\
\text { aneurysms }\end{array}$ & $8(36 \%)$ & $47(12 \%)$ & 0.004 \\
\hline $\begin{array}{l}\text { No. of pts w/ radiographic } \\
\text { vasospasm* }\end{array}$ & $9 / 17(53 \%)$ & $105 / 212(50 \%)$ & 0.88 \\
\hline $\begin{array}{l}\text { No. of pts w/ clinical vaso- } \\
\text { spasm }^{*}\end{array}$ & $2 / 17(12 \%)$ & $48 / 212(23 \%)$ & 0.38 \\
\hline
\end{tabular}

tion followed by arteriovenous malformation resection. Of the 14 patients with saccular-type aneurysms, 1 died before treatment, and 13 were treated with primary clip occlusion or coil occlusion.

In the microsurgical group, 1 patient with a dissectingtype aneurysm who was treated with clip wrapping had an early postoperative death. Vasospasm data were available for 17 of the 22 patients with PICA aneurysms. Radiographic vasospasm was present in 9 patients (53\%), and 2 patients (12\%) experienced clinical vasospasm. Vasospasm data for 212 non-PICA aneurysm patients enrolled in the BRAT were available for comparison: $105(50 \%)$ of these patients had radiographic vasospasm, and 48 (23\%) had clinical vasospasm. There was no statistically significant difference in the rates of radiographic or clinical vasospasm between PICA patients and non-PICA patients (Table 1).

At the time of discharge, 20 patients (91\%) with ruptured PICA aneurysms were considered to have a poor outcome (mRS score $>2)$. At 1 and 3 years after treatment, 3 patients were lost to follow-up and 12 patients $(63 \%)$ were considered to have a poor outcome. Of the 20 patients who were discharged from the hospital, $10(50 \%)$ had both a tracheostomy and a PEG tube placed due to persistent lower cranial nerve dysfunction. In comparison with the remainder of the patients with ruptured aneurysms in the BRAT, patients with ruptured PICA aneurysms had a significantly higher chance of tracheostomy/ PEG tube placement at discharge (50\% vs $16 \%$, $\mathrm{p}<0.001)$. Neither of the patients with a ruptured PICA aneurysm treated with coiling underwent tracheostomy/PEG tube placement. In the remainder of the BRAT cohort, however, there was no significant difference in the rate of trache-
ostomy/PEG tube placement between those treated with clipping versus coiling $(19.5 \%$ vs $13.5 \%, \mathrm{p}=0.22)$. There was no difference in the rate of tracheostomy/PEG placement between patients with proximal saccular PICA aneurysms and those with distal dissecting type aneurysms (50\% vs $50 \%)$. In addition, patients with ruptured PICA aneurysms had significantly poorer outcomes at discharge and at 1- and 3-year follow-up compared with all other ruptured aneurysm patients (91\% vs $67 \%$ at discharge, $\mathrm{p}=$ $0.017 ; 63 \%$ vs $29 \%$ at 1 year, $p=0.002 ; 63 \%$ vs $32 \%$ at 3 years, $\mathrm{p}=0.006)$ (Table 2 ).

\section{Discussion}

Ruptured PICA aneurysms, although relatively uncommon compared with other ruptured intracranial aneurysms, can be a challenging entity to treat and manage. The PICA can have variable anatomy in both its origin from the vertebral artery and its course through the lower cranial nerves. ${ }^{10,16}$ In addition, PICA aneurysms arise in a variable pattern from the parent vessel, both proximally and distally, and proximal PICA aneurysms are often intimately involved in the lower cranial nerve rootlets.

Our data suggest that patients with ruptured PICA aneurysms have worse outcomes than patients with ruptured aneurysms in other locations. These outcomes were not explained by a difference in Fisher grade, Hunt and Hess grade, or vasospasm rate at admission. We hypothesized that the difference in outcomes was likely due to the high rate of lower cranial nerve dysfunction seen in these patients who ultimately required tracheostomy and PEG tube placement. We found that PICA patients were much more likely to have persistent lower cranial nerve dysfunction requiring intervention compared with the remainder of the BRAT cohort.

We also found that PICA aneurysms were more likely to be fusiform in nature compared with aneurysms in other locations. In comparison with saccular aneurysms, fusiform aneurysms can be much more difficult to treat. Because with fusiform aneurysms a length of the vessel is diseased, treatment often requires clip reconstruction of the vessel, clip wrapping of the vessel, or bypass. The increased operating time as well as the unstable nature of these lesions certainly may help to explain the worse outcome seen in our PICA aneurysm cohort.

This high rate of lower cranial nerve dysfunction is likely multifactorial. The large clot burden often seen

TABLE 2. Patient outcomes

\begin{tabular}{|c|c|c|c|}
\hline Characteristic & $\begin{array}{l}\text { Pts w/ PICA } \\
\text { Aneurysms }\end{array}$ & $\begin{array}{l}\text { Pts w/ Non-PICA } \\
\text { Aneurysms }\end{array}$ & $p$ Value $^{*}$ \\
\hline $\begin{array}{l}\text { Tracheostomy \&/or PEG } \\
\text { tube placement } †\end{array}$ & $10 / 20(50 \%)$ & $59 / 379(16 \%)$ & $<0.001$ \\
\hline $\begin{array}{l}\text { Poor outcome at dis- } \\
\text { charge } \neq\end{array}$ & $20 / 22(91 \%)$ & $259 / 389(67 \%)$ & 0.017 \\
\hline Poor outcome at 1 yr $\ddagger$ & $12 / 19(63 \%)$ & $99 / 344(29 \%)$ & 0.002 \\
\hline Poor outcome at 3 yrs $\ddagger$ & $12 / 19(63 \%)$ & $108 / 335(32 \%)$ & 0.006 \\
\hline
\end{tabular}


at surgery compressing the brainstem and lower cranial nerves is likely a major factor. Open surgical treatment often requires some manipulation of the lower cranial nerves to adequately expose and clip these aneurysms. However, if direct surgical manipulation of the lower cranial nerves were a major factor, then one would expect the tracheostomy/PEG rate to be lower in patients with dissecting aneurysms, which was not the case in our patient population. The high rate of lower cranial nerve dysfunction with PICA aneurysms has been previously reported; Al-khayat et al. found an almost 50\% rate of lower cranial nerve palsy in patients treated for vertebral artery and PICA aneurysms, with those patients having a higher rate of nosocomial pneumonia and longer ICU stay. ${ }^{1}$ Similarly, Horowitz et al. reported a $66 \%$ rate of new postsurgical neurological deficits in a series of 38 treated patients. ${ }^{9}$ The higher rate of nosocomial pneumonia and longer ICU stay can certainly help to explain why patients with ruptured PICA aneurysms would fare worse than patients with ruptured aneurysms in other locations.

The techniques and outcomes of both microsurgical clipping and endovascular coiling have been described; however, most of the reports are retrospective in nature and most do not report long-term outcomes. Also, the rates of good outcomes and morbidity reported in the literature are highly variable. Rodríguez-Hernández and Lawton reported their institution's results in 50 patients with PICA aneurysms treated with microsurgical clipping. With a mean follow-up of $11 \pm 9.9$ months, $80 \%$ of patients experienced a good outcome (Glasgow Outcome Scale [GOS] score 4 or 5). However, it is unclear how many of these patients with aneurysms presented with SAH. ${ }^{16}$ Mericle et al. reviewed 31 consecutive cases of proximal PICA aneurysms treated with endovascular coiling. They were able to achieve excellent angiographic occlusion in $97 \%$ of patients. With an average follow-up of 10 months, $68 \%$ of patients had good outcomes (GOS score 4 or 5). All of these patients presented with SAH..$^{13}$ Peluso et al. also reported their large experience of 46 patients with PICA aneurysms treated with endovascular coiling. They achieved complete or near complete angiographic occlusion in $83 \%$ of cases but had a $19 \%$ procedural rupture rate and a $4.3 \%$ mortality rate. In this group, $80 \%$ of the patients presented with SAH, and at 6 months 35 of the 38 surviving patients $(92 \%)$ had a good outcome.${ }^{15}$ Crowley et al. found that in a cohort of 13 patients with ruptured PICA aneurysms treated with endovascular coiling, $66 \%$ of patients had a good outcome at last follow-up. ${ }^{6}$ Coert et al. in 2007 reviewed their experience with posterior circulation fusiform aneurysms treated both surgically and with endovascular coiling. In this study, they did not differentiate between PICA and vertebral artery aneurysms. They did find at an average follow-up of 33 months that $62 \%$ of the patients with PICA/vertebral artery aneurysms had a good outcome (GOS score 4 or 5). It was not specified exactly how many of the PICA/vertebral artery aneurysm patients presented with SAH or how many were treated with clipping versus coiling. ${ }^{5}$ Chalouhi et al. reviewed 76 cases involving patients who underwent endovascular treatment for either proximal or distal PICA aneurysms. They achieved complete occlusion in only $63 \%$ of the pa- tients but had favorable outcomes in $93 \%$ of the patients with unruptured aneurysms and $78 \%$ of those with ruptured aneurysms. ${ }^{4}$ Finally, Horiuchi et al. described their experience of 24 patients with distal PICA aneurysms; 23 of these patients presented with SAH. Employing a variety of surgical and endovascular techniques, the authors achieved a good outcome in $83 \%$ of patients with an unspecified length of follow-up. ${ }^{8}$

In the BRAT, $91 \%$ of patients with ruptured PICA aneurysms had a poor outcome at discharge; however, this did improve to $63 \%$ at 1- and 3-year follow-up. Our data certainly paint a much more pessimistic picture of the long-term outcome for patients with ruptured PICA aneurysms than previously published studies. However, our data represent the first prospective collection of data for patients with ruptured PICA aneurysms and long-term follow-up.

Of important note is the fact that the randomization scheme failed to adequately randomize patients with ruptured PICA aneurysms between either clipping or coiling in the BRAT. Only 3 patients with PICA aneurysms were initially assigned to the coiling group, and one of these patients crossed over to the clipping group, while the other 18 were assigned to the clipping group (1 patient died before treatment). The 2 patients who were treated with endovascular coil occlusion did fare better than the remainder of the PICA cohort; neither required a tracheostomy or feeding tube and both had an mRS score of 1 at 1-year follow-up. However, a direct comparison with microsurgical clipping is inappropriate, given the small number of patients treated with coiling. A larger prospective trial would be required to determine if there is a difference in outcome among patients with PICA aneurysms treated with microsurgical clipping or endovascular coiling.

Limitations of this study include the retrospective nature of the vasospasm data collection and the small number of patients with ruptured PICA aneurysms who were treated. The placement of a tracheostomy and/or PEG tube was used as a surrogate marker for lower cranial nerve dysfunction. However, the need for a tracheostomy and/or PEG tube is multifactorial; a persistent depressed level of consciousness and pulmonary disease certainly can play a role. We were unable to discern from the clinical charts what the primary reason for tracheostomy and/or PEG tube placement was in most patients. Without a true measure of lower cranial nerve dysfunction, the placement of tracheostomy and/or PEG tubes seemed to be the most appropriate outcome for evaluation, albeit an imperfect one.

\section{Conclusions}

PICA aneurysms are relatively rare cerebral aneurysms requiring treatment. In this cohort of SAH patients with ruptured PICA aneurysms treated as part of a large, randomized, prospective trial, the rate of lower cranial nerve dysfunction, as measured by tracheostomy and/or PEG tube placement, was significantly higher than the rate for patients with all other ruptured aneurysms $(50 \%$ vs $16 \%, \mathrm{p}$ $<0.001)$. In addition, the PICA aneurysms were more likely to be fusiform (36\% vs $12 \%, \mathrm{p}=0.004)$. Furthermore, patients with ruptured PICA aneurysms had significantly 
worse outcomes at discharge and at 1- and 3-year followup than patients with non-PICA aneurysms. Both the high rate of lower cranial nerve dysfunction and the high rate of fusiform aneurysms in this cohort may help to explain this poor outcome.

\section{References}

1. Al-khayat H, Al-Khayat H, Beshay J, Manner D, White J: Vertebral artery-posteroinferior cerebellar artery aneurysms: clinical and lower cranial nerve outcomes in 52 patients. Neurosurgery 56:2-11, 2005

2. Bradac GB, Bergui M: Endovascular treatment of the posterior inferior cerebellar artery aneurysms. Neuroradiology 46:1006-1011, 2004

3. Cellerini M, Mangiafico S, Ammannati F, Ambrosanio G, Muto M, Galasso L, et al: Ruptured, dissecting posterior inferior cerebellar artery aneurysms: endovascular treatment without parent vessel occlusion. Neuroradiology 50:315320,2008

4. Chalouhi N, Jabbour P, Starke RM, Tjoumakaris SI, Gonzalez LF, Witte S, et al: Endovascular treatment of proximal and distal posterior inferior cerebellar artery aneurysms. J Neurosurg 118:991-999, 2013

5. Coert BA, Chang SD, Do HM, Marks MP, Steinberg GK: Surgical and endovascular management of symptomatic posterior circulation fusiform aneurysms. J Neurosurg 106: $855-865,2007$

6. Crowley RW, Albuquerque FC, Ducruet AF, Williamson RW, McDougall CG: Technical considerations in the endovascular management of aneurysms of the posterior inferior cerebellar artery. Neurosurgery 71 (2 Suppl Operative):ons204ons218, 2012

7. D'Ambrosio AL, Kreiter KT, Bush CA, Sciacca RR, Mayer SA, Solomon RA, et al: Far lateral suboccipital approach for the treatment of proximal posteroinferior cerebellar artery aneurysms: surgical results and long-term outcome. Neurosurgery 55:39-54, 2004

8. Horiuchi T, Tanaka Y, Hongo K, Nitta J, Kusano Y, Kobayashi S: Characteristics of distal posteroinferior cerebellar artery aneurysms. Neurosurgery 53:589-596, 2003

9. Horowitz M, Kopitnik T, Landreneau F, Krummerman J, Batjer HH, Thomas G, et al: Posteroinferior cerebellar artery aneurysms: surgical results for 38 patients. Neurosurgery 43:1026-1032, 1998
10. Hudgins RJ, Day AL, Quisling RG, Rhoton AL Jr, Sypert GW, Garcia-Bengochea F: Aneurysms of the posterior inferior cerebellar artery. A clinical and anatomical analysis. J Neurosurg 58:381-387, 1983

11. Lewis SB, Chang DJ, Peace DA, Lafrentz PJ, Day AL: Distal posterior inferior cerebellar artery aneurysms: clinical features and management. J Neurosurg 97:756-766, 2002

12. McDougall CG, Spetzler RF, Zabramski JM, Partovi S, Hills NK, Nakaji P, et al: The Barrow Ruptured Aneurysm Trial. J Neurosurg 116:135-144, 2012

13. Mericle RA, Reig AS, Burry MV, Eskioglu E, Firment CS, Santra S: Endovascular surgery for proximal posterior inferior cerebellar artery aneurysms: an analysis of Glasgow Outcome Score by Hunt-Hess grades. Neurosurgery 58:619-625, 2006

14. Pandey AS, Koebbe C, Rosenwasser RH, Veznedaroglu E: Endovascular coil embolization of ruptured and unruptured posterior circulation aneurysms: review of a 10 -year experience. Neurosurgery 60:626-637, 2007

15. Peluso JP, van Rooij WJ, Sluzewski M, Beute GN, Majoie $\mathrm{CB}$ : Posterior inferior cerebellar artery aneurysms: incidence, clinical presentation, and outcome of endovascular treatment. AJNR Am J Neuroradiol 29:86-90, 2008

16. Rodríguez-Hernández A, Lawton MT: Anatomical triangles defining surgical routes to posterior inferior cerebellar artery aneurysms. J Neurosurg 114:1088-1094, 2011

\section{Author Contributions}

Conception and design: Spetzler, Williamson, McDougall, Nakaji, Albuquerque. Acquisition of data: Spetzler, McDougall, Nakaji, Albuquerque. Analysis and interpretation of data: Williamson, Wilson, Abla. Drafting the article: Williamson. Critically revising the article: Spetzler, Wilson, Abla, McDougall, Nakaji. Reviewed submitted version of manuscript: Spetzler. Statistical analysis: Williamson.

\section{Correspondence}

Robert F. Spetzler, c/o Neuroscience Publications, Barrow Neurological Institute, St. Joseph's Hospital and Medical Center, 350 W. Thomas Rd., Phoenix, AZ 85013. email: neuropub@dignity health.org. 